\title{
DYNAMICAL BEHAVIOR OF VOLTERRA MODEL WITH MUTUAL INTERFERENCE CONCERNING IPM*
}

\author{
Yujuan Zhang ${ }^{1,2}$, Bing LiU ${ }^{1}$ and Lansun Chen ${ }^{2}$
}

\begin{abstract}
A Volterra model with mutual interference concerning integrated pest management is proposed and analyzed. By using Floquet theorem and small amplitude perturbation method and comparison theorem, we show the existence of a globally asymptotically stable pest-eradication periodic solution. Further, we prove that when the stability of pest-eradication periodic solution is lost, the system is permanent and there exists a locally stable positive periodic solution which arises from the pest-eradication periodic solution by bifurcation theory. When the unique positive periodic solution loses its stability, numerical simulation shows there is a characteristic sequence of bifurcations, leading to a chaotic dynamics. Finally, we compare the validity of integrated pest management (IPM) strategy with classical methods and conclude IPM strategy is more effective than classical methods.
\end{abstract}

Mathematics Subject Classification. 34A37, 92D25.

Received: July 6, 2003.

\section{INTRODUCTION}

It is well known that pest outbreak can cause great economic loss and pest control has been concerned by entomologist and society. There are many ways to beat agricultural pests. Combining different pest control strategies is the basis of integrated pest management (IPM). It involves chemical, cultural, biological, and mechanical methods in a way that minimizes economic, health, and environmental risks.

Biological control is an important method for pest control. The principle behind biological control is that a given pest has enemies - predators, parasites or pathogens. Beneficial natural enemy plays a more active role in suppressing insect pests. By introducing or encouraging such enemies, the population of pest organisms should decline $[2,5,13,16]$. One approach of biological control is to release beneficial natural enemies to control insect and mite pest. This approach is known as augmentation. The practice of augmentation is based on the idea that, in some situations, there are not adequate numbers or species of natural enemies to provide optimal biological control, but that the numbers can be increased by releases. This approach is a highly efficacious, cost effective and environmentally sound approach to pest management.

Chemical control is another important method for pest control. It forms part of an IPM strategy. Insecticides are useful because they quickly kill a significant portion of an insect population and they sometimes provide the only feasible methods for preventing economic loss. The key is to use pesticides in a way that complements

Keywords and phrases. Integrated pest management (IPM), mutual interference, permanence, bifurcation, chaos.

* Integrated pest management.

1 Department of Mathematics, Anshan Normal University, Anshan, Liaoning 114005, P.R. China.

e-mail: yujuanz2000@yahoo.com

2 Department of Applied Mathematics, Dalian University of Technology, Dalian, Liaoning 116024, P.R. China. 
rather than hinders other elements in the strategy. For example, when the size of enemies is too small to control pests or the cost of controlling pest is too high, pesticide has to be used. It is important to understand the life cycle of pest so that the pesticide can be applied when the pest is at its most vulnerable - the aim is to achieve maximum effect at minimum levels of pesticide.

Whenever possible, different pest control techniques should work together rather than against each other. In some cases, this can lead to synergy - where the combined effect of different techniques is greater than would be expected from simply adding the individual effects together. It has been proved IPM strategy is more effective than the classical methods (only biological control or chemical control) $[12,18,19]$.

The main purpose of this paper is to construct a simple mathematical model according to the fact of IPM and investigate the dynamics of this system. We suggest an impulsive system to model the process of periodic releasing natural enemies and spraying pesticide (or harvesting pest) at fixed moment in next section. In Section 3, we will give some notations and lemmas. By using the Floquet theory of impulsive differential equation, small amplitude perturbation method and comparison theorem, we consider the globally asymptotic stability of pest-eradication periodic solution and give the condition for the permanence of the system in Section 4. In Section 5, we show the existence of locally stable positive periodic solution by bifurcation theory. Further numerical results in Section 6 imply that the system exhibit complicated dynamical behaviors. In last section, we conclude with a brief discussion and comparison of validity of IPM strategy with classical methods.

\section{Model FORMUlation}

When predators (parasites) search for prey (hosts), they interfere each other. Part of this interference results in dispersion of the parasites. Most data from laboratory experiments show that the searching efficiency per individual decreases as parasite density increase - resulting in a reduced time spent uninterruptedly probing for hosts and consequently more time "wasted" in walking, resting and cleaning. Hassell reviewed some of these example in [8]. A further example, that of Trichogramma evanescens attacking eggs of Sitotroga cereallela (Oliv.) was given by Edwards in [4]. The model concerning the relationship of the mutual interference of this kind with the density of parasite was proposed by Hassell and Varley [9], and further studied by Hassell, Rogers, et al. $[7,8,10,17]$. They based their model on measurements of the outcome of search by known parasite populations, and showed interference to be an important component. The model we considered is based on the following Volterra model with mutual interference which was investigated by $\mathrm{Wu}$ [20].

$$
\left\{\begin{array}{l}
\frac{\mathrm{d} x_{1}}{\mathrm{~d} t}=x_{1}\left(a_{10}-a_{11} x_{1}-a_{12} x_{2}^{m}\right) \\
\frac{\mathrm{d} x_{2}}{\mathrm{~d} t}=x_{2}\left(-a_{20}+k a_{12} x_{1} x_{2}^{m-1}\right)
\end{array}\right.
$$

where $x_{1}(t)$ is the density of prey (pest), $x_{2}(t)$ is the density of predator (natural enemy). $0 \leq m<1$ is interference constant. $a_{10}, a_{11}, a_{12}, a_{20}$ and $k$ are positive constants. There exist three equilibria for system (2.1), namely $E_{1}(0,0), E_{2}\left(\frac{a_{10}}{a_{20}}, 0\right)$ and positive equilibrium $E_{3}\left(x_{1}^{*}, x_{2}^{*}\right)$. Where $E_{1}$ and $E_{2}$ are saddle points, $E_{3}$ is globally asymptotically stable. Thus, we can see that there is no stable pest-eradication equilibrium. Therefore, using this method to suppress pests is not effective. Now, we develop model (2.1) by introducing periodic release of predator and spraying of pesticide. The model is described by the following system:

$$
\left\{\begin{aligned}
\frac{\mathrm{d} x_{1}}{\mathrm{~d} t} & =x_{1}\left(a_{10}-a_{11} x_{1}-a_{12} x_{2}^{m}\right), \\
\frac{\mathrm{d} x_{2}}{\mathrm{~d} t} & =x_{2}\left(-a_{20}+k a_{12} x_{1} x_{2}^{m-1}\right),
\end{aligned}\right\} \quad t \neq n \tau,
$$


where $\triangle x_{1}(t)=x_{1}\left(t^{+}\right)-x_{1}(t), \triangle x_{2}(t)=x_{2}\left(t^{+}\right)-x_{2}(t), \tau$ is the period of the impulse which could be artificially planting in order to eradicate pests to prevent increasing pest populations from causing an economic loss. $n \in Z_{+}, Z_{+}=\{1,2, \cdots\}, 0 \leq c<1$ is the proportion of pest reduced by catching or spraying of pesticide, $d>0$ is the number of predators released each time. We can use a combination of biological (natural enemies), cultural (catching) and chemical (killing) tactics to eradicate pests, and show the efficiency of IPM strategy.

\section{Preliminaries}

In this section, we will give some definitions, notations and some lemmas which will be useful for our main results.

Let $R_{+}=[0, \infty), R_{+}^{2}=\left\{x \in R^{2}: x>0\right\}$. Denote $f=\left(f_{1}, f_{2}\right)$ the map defined by the right hand of the first two equations of the system (2.2). Let $V: R_{+} \times R_{+}^{2} \rightarrow R_{+}$, then $V$ is said to belong to class $V_{0}$ if

(i) $V$ is continuous in $(n \tau,(n+1) \tau] \times R_{+}^{2}$ and for each $x \in R_{+}^{2}, n \in Z_{+}, \lim _{(t, y) \rightarrow\left(n \tau^{+}, x\right)} V(t, y)=V\left(n \tau^{+}, x\right)$ exists;

(ii) $V$ is locally Lipschitzian in $x$.

Definition 3.1. $V \in V_{0}$, then for $(t, x) \in(n \tau,(n+1) \tau] \times R_{+}^{2}$, the upper right derivative of $V(t, x)$ with respect to the impulsive differential system $(2.2)$ is defined as

$$
D^{+} V(t, x)=\lim _{h \rightarrow 0^{+}} \sup \frac{1}{h}[V(t+h, x+h f(t, x))-V(t, x)] .
$$

Definition 3.2. It is said that system (2.2) is permanent if there exist positive constants $\rho<\varrho$ and a finite time $\bar{\tau}$, such that each positive solution $\left(x_{1}(t), x_{2}(t)\right)$ of the system satisfies $\rho \leq x_{i}(t) \leq \varrho, i=1,2$ for all $t>\bar{\tau}$.

The solution of the system (2.2) $x(t): R_{+} \rightarrow R_{+}^{2}$ is continuously differential in $R_{+}-\{n \tau\}, n \in Z_{+}$and $x\left(n \tau^{+}\right)=\lim _{t \rightarrow n \tau^{+}} x(t)$ exists. The smoothness properties of $f$ guarantee the global existence and uniqueness of solutions of the system (2.2). The following lemma is obvious.

Lemma 3.1. Suppose $x(t)$ is a solution of the system (2.2) subject to $x\left(0^{+}\right) \geq 0$, then $x(t) \geq 0$ for all $t \geq 0$.

The next comparison theorem on impulsive differential equation [14] plays an important role:

Lemma 3.2. Let $V: R_{+} \times R^{2} \rightarrow R_{+}$and $V \in V_{0}$. Assume that

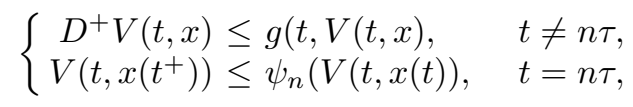

where $g: R_{+} \times R_{+} \rightarrow R$ is continuous in $(n \tau,(n+1) \tau] \times R_{+}$and for $\nu \in R_{+}, n \in Z_{+}, \lim _{(t, y) \rightarrow\left(n \tau^{+}, \nu\right)} g(t, y)=$ $g\left(n \tau^{+}, \nu\right)$ exists, $\psi_{n}: R_{+} \rightarrow R_{+}$is nondecreasing. Let $r(t)$ be the maximal solution of the scalar impulsive differential equation

$$
\left\{\begin{aligned}
\frac{\mathrm{d} u(t)}{\mathrm{d} t}=g(t, u), & & t \neq n \tau, \\
u\left(t^{+}\right)=\psi_{n}(u(t)), & & t=n \tau, \\
u\left(0^{+}\right)=u_{0}, & &
\end{aligned}\right.
$$

existing on $[0, \infty)$. Then $V\left(0^{+}, x_{0}\right) \leq u_{0}$ implies that $V(t, x(t)) \leq r(t), t \geq 0$, where $x(t)$ is any solution of (2.2).

Assume that $V \in V_{0}$, the inequalities in (3.1) are reversed and $\psi_{n}$ is nonincreasing. Let $\rho(t)$ be the minimal solution of $(3.2)$ existing on $[0, \infty)$. Then $V(t, x) \geq \rho(t), t \geq 0$. Note that if we have some smoothness conditions of $g(t)$ to guarantee the existence and uniqueness of solutions for (3.2), then $r(t)$ and $\rho(t)$ are exactly the unique solution of (3.2). 
Finally, we give some basic properties about the following subsystem

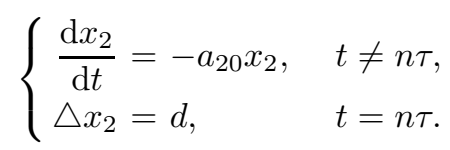

Clearly $\tilde{x}_{2}(t)=\frac{d \exp \left(-a_{20}(t-n \tau)\right)}{1-\exp \left(-a_{20} \tau\right)}\left(t \in(n \tau,(n+1) \tau], n \in Z_{+}, \tilde{x}_{2}\left(0^{+}\right)=\frac{d}{1-\exp \left(-a_{20} \tau\right)}\right)$ is a positive periodic solution of system (3.3). Since the solution of (3.3) with initial value $x_{2}\left(0^{+}\right)=x_{20} \geq 0$ is $x_{2}(t)=\left(x_{2}\left(0^{+}\right)-\right.$ $\left.\frac{d}{1-\exp \left(-a_{20} \tau\right)}\right) \exp \left(-a_{20} t\right)+\tilde{x}_{2}(t), t \in(n \tau,(n+1) \tau], n \in Z_{+}$, we have:

Lemma 3.3. System (3.3) has a positive periodic solution $\tilde{x}_{2}(t)$ and for every solution $x_{2}(t)$ of (3.3) with initial value $x_{2}\left(0^{+}\right)=x_{20} \geq 0$, we have $x_{2}(t) \rightarrow \tilde{x}_{2}(t)$ as $t \rightarrow \infty$.

Therefore, the $\operatorname{system}(2.2)$ has a pest-eradication periodic solution

$$
\left(0, \tilde{x}_{2}(t)\right)=\left(0, \frac{\mathrm{d} \exp \left(-a_{20}(t-n \tau)\right)}{1-\exp \left(-a_{20} \tau\right)}\right)
$$

for $n \tau<t \leq(n+1) \tau$.

\section{Pest-extinction And Permanence of the system}

In this section, we first study the stability of pest-eradication periodic solution.

Theorem 4.1. Let $\left(x_{1}(t), x_{2}(t)\right)$ be any solution of (2.2), then $\left(0, \tilde{x}_{2}(t)\right)$ is globally asymptotically stable provided

$$
(1-c) \exp \left(a_{10} \tau-\frac{a_{12} d^{m}}{a_{20} m} \frac{1-\exp \left(-a_{20} m \tau\right)}{\left(1-\exp \left(-a_{20} \tau\right)\right)^{m}}\right)<1 .
$$

Proof. First, we prove the local stability. The local stability of periodic solution $\left(0, \tilde{x}_{2}(t)\right)$ may be determined by considering the behavior of small amplitude perturbations of the solution. Define $x_{1}(t)=u(t), x_{2}(t)=$ $v(t)+\tilde{x}_{2}(t)$, there may be written

$$
\left(\begin{array}{l}
u(t) \\
v(t)
\end{array}\right)=\Phi(t)\left(\begin{array}{l}
u(0) \\
v(0)
\end{array}\right), \quad 0 \leq t<\tau,
$$

where $\Phi(t)$ is the fundamental matrix of (4.1), which satisfies

$$
\frac{\mathrm{d} \Phi(t)}{\mathrm{d} t}=\left(\begin{array}{cc}
a_{10}-a_{12} \tilde{x}_{2}^{m} & 0 \\
k a_{12} \tilde{x}_{2}^{m} & -a_{20}
\end{array}\right) \Phi(t)
$$

and $\Phi(0)=I$, the identity matrix. The linearization of $(2.2)$ from the third to the fourth becomes

$$
\left(\begin{array}{l}
u\left(n \tau^{+}\right) \\
v\left(n \tau^{+}\right)
\end{array}\right)=\left(\begin{array}{cc}
1-c & 0 \\
0 & 1
\end{array}\right)\left(\begin{array}{l}
u(n \tau) \\
v(n \tau)
\end{array}\right) .
$$

The stability of the periodic solution $\left(0, \tilde{x}_{2}(t)\right)$ is determined by the eigenvalues of

$$
M=\left(\begin{array}{cc}
1-c & 0 \\
0 & 1
\end{array}\right) \Phi(\tau)
$$


which are $\mu_{1}=\exp \left(-a_{20} \tau\right)<1, \mu_{2}=(1-c) \exp \left(\int_{0}^{\tau}\left(a_{10}-a_{12} \tilde{x}_{2}^{m}\right) \mathrm{d} t\right)$. According to Floquet theory of impulsive differential equation, $\left(0, \tilde{x}_{2}(t)\right)$ is locally stable if and only if $\left|\mu_{2}\right|<1$, i.e., $(1-c) \exp \left(a_{10} \tau-\right.$ $\frac{a_{12} d^{m}}{a_{20} m} \frac{1-\exp \left(-a_{20} m \tau\right)}{\left.\left(1-\exp \left(-a_{20} \tau\right)\right)^{m}\right)}<1$.

In the following, we prove the global attractivity. Choose a $\varepsilon>0$ small enough such that

$$
\sigma \triangleq(1-c) \exp \left(\int_{0}^{\tau}\left(a_{10}-a_{12} \tilde{x}_{2}^{m}+a_{12} m \tilde{x}_{2}^{m-1} \varepsilon\right) \mathrm{d} t\right)<1
$$

Note that $\frac{\mathrm{d} x_{2}}{\mathrm{~d} t} \geq-a_{20} x_{2}$, consider the following impulsive differential equation:

$$
\left\{\begin{aligned}
\frac{\mathrm{d} z}{\mathrm{~d} t} & =-a_{20} z, \quad t \neq n \tau \\
\triangle z & =d, \quad t=n \tau \\
z\left(0^{+}\right) & =x_{2}\left(0^{+}\right) \geq 0
\end{aligned}\right.
$$

From Lemmas 3.2 and 3.3 we have

$$
x_{2}(t) \geq z(t)>\tilde{x}_{2}(t)-\varepsilon,
$$

for $t$ large enough. For simplification we may assume (4.4) holds for all $t \geq 0$. From (2.2) we get

$$
\left\{\begin{array}{l}
\frac{\mathrm{d} x_{1}}{\mathrm{~d} t} \leq x_{1}\left(a_{10}-a_{12} \tilde{x}_{2}^{m}+a_{12} m \tilde{x}_{2}^{m-1} \varepsilon\right), \quad t \neq n \tau, \\
\triangle x_{1}=-c x_{1}, \quad t=n \tau .
\end{array}\right.
$$

Integrate $(4.5)$ on $(n \tau,(n+1) \tau]$, which yields

$$
\begin{aligned}
x_{1}((n+1) \tau) & \leq x_{1}(n \tau)(1-c) \exp \left(\int_{n \tau}^{(n+1) \tau}\left(a_{10}-a_{12} \tilde{x}_{2}^{m}+a_{12} m \tilde{x}_{2}^{m-1} \varepsilon\right) \mathrm{d} t\right) \\
& =x_{1}(n \tau) \sigma .
\end{aligned}
$$

Thus $x_{1}(n \tau) \leq x_{1}\left(0^{+}\right) \sigma^{n}$ and $x_{1}(n \tau) \rightarrow 0$ as $n \rightarrow \infty$. Therefore $x_{1}(t) \rightarrow 0$ as $n \rightarrow \infty$ since $0<x_{1}(t) \leq$ $x_{1}(n \tau)(1-c) \exp \left(a_{10} \tau\right)$ for $n \tau<t \leq(n+1) \tau$.

Next, we prove that $x_{2}(t) \rightarrow \tilde{x}_{2}(t)$ as $t \rightarrow \infty$ if $\lim _{t \rightarrow \infty} x_{1}(t)=0$. Let $m_{2}=\frac{d e^{-a_{20} \tau}}{1-e^{-a_{20} \tau}}-\varepsilon>0, \varepsilon>0$ is small enough. We have proved $x_{2}(t)>\tilde{x}_{2}(t)-\varepsilon$ for $t$ large enough. It is easy to get $x_{2}(t) \geq m_{2}$ for $t$ large enough. We may assume that $x_{2}(t) \geq m_{2}$ for all $t \geq 0$. For $0<\varepsilon_{1}<\frac{a_{20} m_{2}^{1-m}}{k a_{12}}$, there exists a $\tau_{0}>0$ such that $0<x_{1}(t)<\varepsilon_{1}$ for all $t>\tau_{0}$. Assume that $0<x_{1}(t)<\varepsilon_{1}$ for $t \geq 0$. Then we have

$$
-a_{20} x_{2} \leq \frac{\mathrm{d} x_{2}}{\mathrm{~d} t} \leq x_{2}\left(-a_{20}+k a_{12} \varepsilon_{1} m_{2}^{m-1}\right) .
$$

By Lemmas 3.2 and 3.3 we obtain $z(t) \leq x_{2}(t) \leq \omega(t), z(t) \rightarrow \tilde{x}_{2}(t)$ and $\omega(t) \rightarrow \tilde{\omega}(t)$ as $t \rightarrow \infty$, where $z(t)$ and $\omega(t)$ are solutions of $(4.3)$ and

$$
\left\{\begin{aligned}
\frac{\mathrm{d} \omega}{\mathrm{d} t} & =\omega\left(-a_{20}+k a_{12} \varepsilon_{1} m_{2}^{m-1}\right), \quad t \neq n \tau \\
\triangle \omega & =d, \quad t=n \tau \\
\omega\left(0^{+}\right) & =x_{2}\left(0^{+}\right) \geq 0
\end{aligned}\right.
$$

respectively, $\tilde{\omega}(t)=\frac{d \exp \left(\left(-a_{20}+k a_{12} \varepsilon_{1} m_{2}^{m-1}\right)(t-n \tau)\right)}{1-\exp \left(\left(-a_{20}+k a_{12} \varepsilon_{1} m_{2}^{m-1}\right) \tau\right)}, n \tau<t \leq(n+1) \tau$. Therefore, for any $\varepsilon_{2}>0$, there exists $\tau_{1}>0$ such that $\tilde{x}_{2}(t)-\varepsilon_{2}<x_{2}(t)<\tilde{\omega}(t)+\varepsilon_{2}$ for $t>\tau_{1}$. Let $\varepsilon_{1} \rightarrow 0$, we get $\tilde{x}_{2}(t)-\varepsilon_{2}<x_{2}(t)<\tilde{x}_{2}(t)+\varepsilon_{2}$. Hence $x_{2}(t) \rightarrow \tilde{x}_{2}(t)$ as $t \rightarrow \infty$. This completes the proof. 
In order to consider the permanence of the system, we will show that all solutions of (2.2) are uniformly ultimately bounded.

Theorem 4.2. There exists a constant $M>0$ such that $x_{i}(t) \leq M, i=1,2$ for each positive solution $x(t)=\left(x_{1}(t), x_{2}(t)\right)$ of (2.2) with $t$ large enough.

Proof. Define function $V(t)=k x_{1}(t)+x_{2}(t)$. When $t \neq n \tau$, choose $0 \leq \lambda \leq a_{20}$, we have

$$
D^{+} V(t)+\lambda V(t)=-k a_{11} x_{1}^{2}(t)+k a_{10} x_{1}(t)+\lambda k x_{1}(t)+\left(\lambda-a_{20}\right) x_{2}(t) \leq K,
$$

where $K=\frac{k\left(\lambda+a_{10}\right)^{2}}{4 a_{11}}$. When $t=n \tau$,

$$
V\left(n \tau^{+}\right)=k(1-c) x_{1}(n \tau)+x_{2}(n \tau)+d \leq V(n \tau)+d .
$$

According to Lemma 2.2 in [1], for $t \in(n \tau,(n+1) \tau]$, we have

$$
\begin{aligned}
V(t) & =V(0) \mathrm{e}^{-\lambda t}+\int_{0}^{t} K \mathrm{e}^{-\lambda(t-s)} \mathrm{d} s+\sum_{0<i \tau<t} d \mathrm{e}^{-\lambda(t-i \tau)} \\
& \leq V(0) \mathrm{e}^{-\lambda t}+\frac{K}{\lambda}\left(1-\mathrm{e}^{-\lambda t}\right)+\frac{\mathrm{d} e^{-\lambda(t-\tau)}}{1-\mathrm{e}^{\lambda \tau}}+\frac{d \mathrm{e}^{\lambda \tau}}{\mathrm{e}^{\lambda \tau}-1} \\
& \rightarrow \frac{K}{\lambda}+\frac{d \mathrm{e}^{\lambda \tau}}{\mathrm{e}^{\lambda \tau}-1}, \quad t \rightarrow \infty .
\end{aligned}
$$

So by the definition of $V(t)$ we obtain that each positive solution of the system (2.2) is uniformly ultimately bounded. Hence, there exists a constant $M>0$ such that $x_{i}(t) \leq M, i=1,2$ for $t$ large enough. This completes the proof.

Then, we investigate the permanence of system (2.2).

Theorem 4.3. System (2.2) is permanent provided

$$
(1-c) \exp \left(a_{10} \tau-\frac{a_{12} d^{m}}{a_{20} m} \frac{1-\exp \left(-a_{20} m \tau\right)}{\left(1-\exp \left(-a_{20} \tau\right)\right)^{m}}\right)>1 .
$$

Proof. Suppose $x(t)=\left(x_{1}(t), x_{2}(t)\right)$ is a solution of $(2.2)$ with $x\left(0^{+}\right)>0$. We have proved there exist $m_{2}, M>0$, such that $x_{2}(t) \geq m_{2}, x_{i}(t) \leq M, i=1,2$ for $t$ large enough. We may assume $x_{i}(t) \leq M, i=1,2$ for $t \geq 0, M>\left(\frac{a_{10}}{a_{12}}\right)^{\frac{1}{m}}$. In the following, we want to find $m_{1}>0$ such that $x_{1}(t) \geq m_{1}$ for $t$ large enough. We will do it in the following two steps for convenience.

Step I. From the condition of the theorem, we can choose $0<m_{3}<\frac{a_{20}}{k a_{12}}, \varepsilon>0$ be small enough such that $\delta \triangleq(1-c) \exp \left(a_{10} \tau-a_{11} m_{3} \tau+a_{12} \tau-a_{12}(1+\varepsilon)^{m} \tau+\frac{a_{12} d^{m}}{m\left(-a_{20}+k a_{12} m_{3}\right)} \frac{1-\exp \left(m\left(-a_{20}+k a_{12} m_{3}\right) \tau\right)}{\left(1-\exp \left(\left(-a_{20}+k a_{12} m_{3}\right) \tau\right)\right)^{m}}\right)>1$. We will prove there exists a $t_{1} \in(0, \infty)$ such that $x_{1}\left(t_{1}\right) \geq m_{3}$. Otherwise,

$$
\left\{\begin{array}{l}
\frac{\mathrm{d} x_{2}}{\mathrm{~d} t} \leq x_{2}\left(-a_{20}+k a_{12} m_{3}\right), \quad t \neq n \tau, \\
\triangle x_{2}=d, \quad t=n \tau .
\end{array}\right.
$$

From Lemmas 3.1 and 3.2, we have $x_{2}(t) \leq \nu(t)$ and $\nu(t) \rightarrow \tilde{\nu}(t)$ as $t \rightarrow \infty$, where $\nu(t)$ is the solution of

$$
\left\{\begin{aligned}
\frac{\mathrm{d} \nu}{\mathrm{d} t} & =\nu\left(-a_{20}+k a_{12} m_{3}\right), \quad t \neq n \tau, \\
\triangle \nu & =d, \quad t=n \tau, \\
\nu\left(0^{+}\right) & =x_{2}\left(0^{+}\right) \geq 0
\end{aligned}\right.
$$


$\tilde{\nu}(t)=\frac{d \exp \left(\left(-a_{20}+k a_{12} m_{3}\right)(t-n \tau)\right)}{1-\exp \left(\left(-a_{20}+k a_{12} m_{3}\right) \tau\right)}, t \in(n \tau,(n+1) \tau]$. Therefore, there exists a $\tau_{2}>0$ such that $x_{2}(t) \leq \nu(t)<$ $\tilde{\nu}(t)+\varepsilon$ and

$$
\left\{\begin{array}{l}
\frac{\mathrm{d} x_{1}}{\mathrm{~d} t} \geq x_{1}\left(a_{10}-a_{11} m_{3}-a_{12}(\tilde{\nu}+\varepsilon)^{m}\right), \quad t \neq n \tau, \\
\triangle x_{1}=-c x_{1}, \quad t=n \tau,
\end{array}\right.
$$

for $t>\tau_{2}$. Let $N \in Z_{+}$and $N_{1} \tau \geq \tau_{2}$. Integrating (4.8) on $(n \tau,(n+1) \tau], n \geq N$, we have

$$
\begin{aligned}
x_{1}((n+1) \tau) & \geq x_{1}(n \tau)(1-c) \exp \left(\int_{n \tau}^{(n+1) \tau}\left(a_{10}-a_{11} m_{3}-a_{12}(\tilde{\nu}+\varepsilon)^{m}\right) \mathrm{d} t\right) \\
& \geq x_{1}(n \tau) \delta .
\end{aligned}
$$

Then $x_{1}((N+j) \tau) \geq x_{1}(N \tau) \delta^{j} \rightarrow \infty$, as $j \rightarrow \infty$, which is a contradiction to the boundedness of $x_{1}(t)$. Hence there exists a $t_{1}>0$ such that $x_{1}\left(t_{1}\right) \geq m_{3}$.

Step II. If $x_{1}(t) \geq m_{3}$ for all $t \geq t_{1}$, then our aim is obtained. Otherwise, $x_{1}(t)<m_{3}$ for some $t \geq t_{1}$. Setting $t^{*}=\inf _{t>t_{1}}\left\{x_{1}(t)<m_{3}\right\}$, there are two possible cases for $t^{*}$.

Case (i). $t^{*}=n_{1} \tau, n_{1} \in Z_{+}$. Then $x_{1}(t) \geq m_{3}$ for $t \in\left[t_{1}, t^{*}\right]$ and $(1-c) m_{3} \leq x_{1}\left(t^{*+}\right)=(1-c) x_{1}\left(t^{*}\right) \leq m_{3}$. Choose $n_{2}, n_{3} \in Z_{+}$such that

$$
\begin{gathered}
n_{2} \tau>\frac{1}{-a_{20}+k a_{12} m_{3}} \ln \frac{\varepsilon}{M+d}, \\
(1-c)^{n_{2}} \delta^{n_{3}} \exp \left(n_{2} \alpha \tau\right)>(1-c)^{n_{2}} \delta^{n_{3}} \exp \left(\left(n_{2}+1\right) \alpha \tau\right)>1,
\end{gathered}
$$

where $\alpha=a_{10}-a_{11} m_{3}-a_{12} M^{m}<0$. Let $\tau^{\prime}=n_{2} \tau+n_{3} \tau$, we claim that there must be a $t^{\prime} \in\left(t^{*}, t^{*}+\tau^{\prime}\right]$ such that $x_{1}\left(t^{\prime}\right) \geq m_{3}$. Otherwise, consider (4.7) with $\nu\left(t^{*+}\right)=x_{2}\left(t^{*+}\right)$, we have

$$
\nu(t)=\left(\nu\left(t^{*+}\right)-\frac{d}{1-\exp \left(\left(-a_{20}+k a_{12} m_{3}\right) \tau\right)}\right) \exp \left(\left(-a_{20}+k a_{12} m_{3}\right)\left(t-t^{*}\right)\right)+\tilde{\nu}(t),
$$

for $t \in(n \tau,(n+1) \tau], n_{1} \leq n \leq n_{1}+n_{2}+n_{3}$. Then,

$$
|\nu(t)-\tilde{\nu}(t)|<(M+d) \exp \left(\left(-a_{20}+k a_{12} m_{3}\right)\left(t-t^{*}\right)\right)<\varepsilon
$$

and $x_{2}(t) \leq \nu(t) \leq \tilde{\nu}(t)+\varepsilon$ for $t^{*}+n_{2} \tau \leq t \leq t^{*}+\tau^{\prime}$ which implies that (4.8) holds for $t^{*}+n_{2} \tau \leq t \leq t^{*}+\tau^{\prime}$. So as in step I, we have

$$
x_{1}\left(t^{*}+\tau^{\prime}\right) \geq x_{1}\left(t^{*}+n_{2} \tau\right) \delta^{n_{3}} .
$$

From the system (2.2), we get

$$
\left\{\begin{array}{l}
\frac{\mathrm{d} x_{1}}{\mathrm{~d} t} \geq x_{1}\left(a_{10}-a_{11} m_{3}-a_{12} M^{m}\right), \quad t \neq n \tau, \\
\triangle x_{1}=-c x_{1}, \quad t=n \tau,
\end{array}\right.
$$

for $t \in\left[t^{*}, t^{*}+n_{2} \tau\right]$. Integrating (4.10) on $\left[t^{*}, t^{*}+n_{2} \tau\right]$, we have

$$
x_{1}\left(t^{*}+n_{2} \tau\right) \geq m_{3}(1-c)^{n_{2}} \exp \left(n_{2} \alpha \tau\right) .
$$

Thus we have $x_{1}\left(t^{*}+\tau^{\prime}\right) \geq m_{3}(1-c)^{n_{2}} \delta^{n_{3}} \exp \left(n_{2} \alpha \tau\right)>m_{3}$, which is a contradiction. 
Let $\tilde{t}=\inf _{t>t^{*}}\left\{x_{1}(t) \geq m_{3}\right\}$, then for $t \in\left(t^{*}, \tilde{t}\right], x_{1}(t)<m_{3}$ and $x_{1}(\tilde{t})=m_{3}$ since $x_{1}(t)$ is left continuous and $x_{1}\left(t^{+}\right)=(1-c) x_{1}(t) \leq x_{1}(t)$ for $t=n \tau$, thus $\tilde{t}$ can't be impulsive moment. For $t \in\left(t^{*}, \tilde{t}\right)$, suppose $t \in\left(t^{*}+(l-1) \tau, t^{*}+l \tau\right], l \in Z_{+}$and $l \leq n_{2}+n_{3}$, from (4.10) we have

$$
\begin{aligned}
x_{1}(t) & \geq x_{1}\left(t^{*+}\right)(1-c)^{l-1} \exp ((l-1) \alpha \tau) \exp \left(\alpha\left(t-\left(t^{*}+(l-1) \tau\right)\right)\right) \\
& \geq m_{3}(1-c)^{l} \exp (l \alpha \tau) \geq m_{3}(1-c)^{n_{2}+n_{3}} \exp \left(\left(n_{2}+n_{3}\right) \alpha \tau\right) \triangleq m_{1}^{\prime} .
\end{aligned}
$$

The same arguments can be continued since $x_{1}(\tilde{t}) \geq m_{3}$.

Case (ii). $t^{*} \neq n \tau, n \in Z_{+}$. Then $x_{1}(t) \geq m_{3}$ for all $t \in\left[t_{1}, t^{*}\right)$ and $x_{1}\left(t^{*}\right)=m_{3}$, suppose $t^{*} \in\left(n_{1}^{\prime} \tau,\left(n_{1}^{\prime}+\right.\right.$ 1) $\tau), n_{1}^{\prime} \in Z_{+}$. We claim that there must be a $t^{\prime \prime} \in\left(\left(n_{1}^{\prime}+1\right) \tau,\left(n_{1}^{\prime}+1\right) \tau+\tau^{\prime}\right]$ such that $x_{1}\left(t^{\prime \prime}\right) \geq m_{3}$. Otherwise consider (4.7) with $\nu\left(\left(n_{1}^{\prime}+1\right) \tau^{+}\right)=x_{2}\left(\left(n_{1}^{\prime}+1\right) \tau^{+}\right)$, in $(n \tau,(n+1) \tau], n_{1}^{\prime} \leq n \leq n_{1}^{\prime}+n_{2}+n_{3}$. So as in case (i), we have

$$
x_{1}\left(\left(n_{1}^{\prime}+1\right) \tau+\tau^{\prime}\right) \geq x_{1}\left(\left(n_{1}^{\prime}+1+n_{2}\right) \tau\right) \delta^{n_{3}}
$$

for $\left(n_{1}^{\prime}+1\right) \tau+n_{2} \tau \leq t \leq\left(n_{1}^{\prime}+1\right) \tau+\tau^{\prime}$. There are two possible cases for $x_{1}(t)$, for $t \in\left(t^{*},\left(n_{1}^{\prime}+1\right) \tau\right]$.

Case (ii a). $x_{1}(t)<m_{3}$, for $t \in\left(t^{*},\left(n_{1}^{\prime}+1\right) \tau\right]$. Then $x_{1}(t)<m_{3}$, for $t \in\left(t^{*},\left(n_{1}^{\prime}+1+n_{2}\right) \tau\right)$. (4.10) holds on $\left[t^{*},\left(n_{1}^{\prime}+1+n_{2}\right) \tau\right]$, so we have

$$
x_{1}\left(\left(n_{1}^{\prime}+1+n_{2}\right) \tau\right) \geq m_{3}(1-c)^{n_{2}} \exp \left(\alpha\left(n_{2}+1\right) \tau\right) .
$$

Thus $x_{1}\left(\left(n_{1}^{\prime}+1\right) \tau+\tau^{\prime}\right) \geq m_{3}(1-c)^{n_{2}} \delta^{n_{3}} \exp \left(\alpha\left(n_{2}+1\right) \tau\right)>m_{3}$, a contradiction.

Let $\bar{t}=\inf _{t>t^{*}}\left\{x_{1}(t) \geq m_{3}\right\}$, then $x_{1}(t)<m_{3}$ for $t \in\left(t^{*}, \bar{t}\right)$ and $x_{1}(\bar{t})=m_{3}$. For $t \in\left(t^{*}, \bar{t}\right)$, suppose $t \in\left(n_{1}^{\prime} \tau+\left(l^{\prime}-1\right) \tau, n_{1}^{\prime} \tau+l^{\prime} \tau\right], l^{\prime} \in Z_{+}, l^{\prime} \leq 1+n_{2}+n_{3}$, and integrating (4.10) on $\left(t^{*}, \bar{t}\right)$, we have

$$
x_{1}(t) \geq m_{3}(1-c)^{l^{\prime}-1} \exp \left(l^{\prime} \alpha \tau\right) \geq m_{3}(1-c)^{n_{2}+n_{3}} \exp \left(\left(n_{2}+n_{3}+1\right) \alpha \tau\right) \triangleq m_{1} \leq m_{1}^{\prime} .
$$

For $t>\bar{t}$, the same arguments can be continued since $x_{1}(\bar{t}) \geq m_{3}$.

Case (ii b). There exists a $t \in\left(t^{*},\left(n_{1}^{\prime}+1\right) \tau\right)$ such that $x_{1}(t) \geq m_{3}$. Let $\hat{t}=\inf _{t>t^{*}}\left\{x_{1}(t) \geq m_{3}\right\}$, then $x_{1}(t)<m_{3}$ for $t \in\left(t^{*}, \hat{t}\right)$ and $x_{1}(\hat{t})=m_{3}$. For $t \in\left(t^{*}, \hat{t}\right),(4.10)$ holds true, integrating (4.10) on $\left(t^{*}, \hat{t}\right)$, we have

$$
x_{1}(t) \geq x_{1}\left(t^{*}\right) \exp \left(\alpha\left(t-t^{*}\right)\right) \geq m_{3} \exp (\alpha \tau)>m_{1} .
$$

For $t>\hat{t}$, the same arguments can be continued since $x_{1}(\hat{t}) \geq m_{3}$.

Hence $x_{1}(t) \geq m_{1}$ for all $t \geq t_{1}$. The proof is completed.

Remark. Let $g(\tau)=(1-c) \exp \left(a_{10} \tau-\frac{a_{12} d^{m}}{a_{20} m} \frac{1-\exp \left(-a_{20} m \tau\right)}{\left(1-\exp \left(-a_{20} \tau\right)\right)^{m}}\right)-1, \lim _{\tau \rightarrow 0} g(\tau)=-c, \lim _{\tau \rightarrow \infty} g(\tau)=\infty, g^{\prime \prime}(\tau)>0$. Therefore, $g(\tau)=0$ has a unique positive solution, denoted by $\tau_{\max }$. From Theorem 4.1 and Theorem 4.3, we know that pest-eradication period solution $\left(0, \tilde{x}_{2}(t)\right)$ is globally asymptotically stable when $\tau<\tau_{\max }$ and the system is permanent when $\tau>\tau_{\max }$.

\section{Existence AND Stability of POSitive PERIOdiC SOlUtion}

In this section, we deal with the problem of the bifurcation of positive periodic solution of the system (2.2), which arises from pest-eradication periodic solution $\left(0, \tilde{x}_{2}(t)\right)$. To use Theorem 2 in [15], it is convenient for the 
computation to exchange the subscripts of $x_{1}$ and $x_{2}$

$$
\left\{\begin{aligned}
\frac{\mathrm{d} x_{1}}{\mathrm{~d} t} & =x_{1}\left(-a_{20}+k a_{12} x_{2} x_{1}^{m-1}\right), \\
\frac{\mathrm{d} x_{2}}{\mathrm{~d} t} & =x_{2}\left(a_{10}-a_{11} x_{2}-a_{12} x_{1}^{m}\right),
\end{aligned}\right\} \quad t \neq n \tau,
$$

All notations used in this section are same as those in [15], then

$$
\begin{aligned}
F_{1}\left(x_{1}, x_{2}\right) & =x_{1}\left(-a_{20}+k a_{12} x_{2} x_{1}^{m-1}\right), \\
F_{2}\left(x_{1}, x_{2}\right) & =x_{2}\left(a_{10}-a_{11} x_{2}-a_{12} x_{1}^{m}\right), \\
\Theta_{1}\left(x_{1}, x_{2}\right) & =x_{1}+d \\
\Theta_{2}\left(x_{1}, x_{2}\right) & =(1-c) x_{2} \\
\zeta(t) & =\left(x_{s}(t), 0\right)=\left(\tilde{x}_{2}(t), 0\right) .
\end{aligned}
$$

So we can compute that

$$
d_{0}^{\prime}=1-\left(\frac{\partial \Theta_{2}}{\partial x_{2}} \frac{\partial \Phi_{2}}{\partial x_{2}}\right)\left(\tau_{0}, x_{0}\right)=1-(1-c) \exp \left(a_{10} \tau_{0}-\frac{a_{12} d^{m}}{a_{20} m} \frac{1-\exp \left(-a_{20} m \tau_{0}\right)}{\left(1-\exp \left(-a_{20} \tau_{0}\right)\right)^{m}}\right)
$$

where $\tau_{0}$ is the root of $d_{0}^{\prime}=0$.

$$
\begin{aligned}
& a_{0}^{\prime}=1-\left(\frac{\partial \Theta_{1}}{\partial x_{1}} \frac{\partial \Phi_{1}}{\partial x_{1}}\right)\left(\tau_{0}, x_{0}\right)=1-\exp \left(-a_{20} \tau_{0}\right)>0 \\
& b_{0}^{\prime}=-\left(\frac{\partial \Theta_{1}}{\partial x_{1}} \frac{\partial \Phi_{1}}{\partial x_{2}}+\frac{\partial \Theta_{1}}{\partial x_{2}} \frac{\partial \Phi_{2}}{\partial x_{2}}\right)\left(\tau_{0}, x_{0}\right) \\
& =-\frac{k a_{12} d^{m} \exp \left(-a_{20} \tau_{0}\right)}{\left(1-\exp \left(-a_{20} \tau_{0}\right)\right)^{m}} \int_{0}^{\tau_{0}} \exp \left(a_{20} u(1-m)\right) \exp \left(a_{10} u-\frac{a_{12} d^{m}}{a_{20} m} \frac{1-\exp \left(-a_{20} m u\right)}{\left(1-\exp \left(-a_{20} \tau_{0}\right)\right)^{m}}\right) \mathrm{d} u<0, \\
& \frac{\partial^{2} \Phi_{2}\left(\tau_{0}, x_{0}\right)}{\partial \bar{\tau} \partial x_{2}}=\left(a_{10}-a_{12} d^{m} \frac{\exp \left(-a_{20} m \tau_{0}\right)}{\left(1-\exp \left(-a_{20} \tau_{0}\right)\right)^{m}}\right) \exp \left(a_{10} \tau_{0}-\frac{a_{12} d^{m}}{a_{20} m} \frac{1-\exp \left(-a_{20} m \tau_{0}\right)}{\left(1-\exp \left(-a_{20} \tau_{0}\right)\right)^{m}}\right), \\
& \frac{\partial^{2} \Phi_{2}\left(\tau_{0}, x_{0}\right)}{\partial x_{1} \partial x_{2}}=\frac{-a_{12} m d^{m-1}}{a_{20}(1-m)} \exp \left(a_{10} \tau_{0}-\frac{a_{12} d^{m}}{a_{20} m} \frac{1-\exp \left(-a_{20} m \tau_{0}\right)}{\left(1-\exp \left(-a_{20} \tau_{0}\right)\right)^{m}}\right) \frac{\exp \left(a_{20}(1-m) \tau_{0}\right)-1}{\left(1-\exp \left(-a_{20} \tau_{0}\right)\right)^{m-1}}<0, \\
& \frac{\partial^{2} \Phi_{2}\left(\tau_{0}, x_{0}\right)}{\partial x_{2}^{2}}=-2 a_{11} \tau_{0} \exp \left(a_{10} \tau_{0}-\frac{a_{12} d^{m}}{a_{20} m} \frac{1-\exp \left(-a_{20} m \tau_{0}\right)}{\left(1-\exp \left(-a_{20} \tau_{0}\right)\right)^{m}}\right)-\frac{k m a_{12}^{2} d^{2 m-1}}{\left(1-\exp \left(-a_{20} \tau_{0}\right)\right)^{2 m-1}} \\
& \times \int_{0}^{\tau_{0}}\left\{\exp \left(a_{10}\left(\tau_{0}-u\right)+\frac{a_{12} d^{m}}{a_{20} m} \frac{\exp \left(-a_{20} m \tau_{0}\right)-\exp \left(-a_{20} m u\right)}{\left(1-\exp \left(-a_{20} \tau_{0}\right)\right)^{m}}\right) \exp \left(-2 a_{20} m u\right)\right. \\
& \left.\times \int_{0}^{u} \exp \left(a_{10} p+a_{20} p-\frac{a_{12} d^{m}}{a_{20} m} \frac{1-\exp \left(-a_{20} m p\right)}{\left(1-\exp \left(-a_{20} \tau_{0}\right)\right)^{m}}\right) \mathrm{d} p\right\} \mathrm{d} u<0 \\
& \frac{\partial \Phi_{1}\left(\tau_{0}, x_{0}\right)}{\partial \bar{\tau}}=\dot{x}_{\zeta}\left(\tau_{0}\right)=-\frac{d a_{20} \exp \left(-a_{20} \tau_{0}\right)}{1-\exp \left(-a_{20} \tau_{0}\right)}<0
\end{aligned}
$$



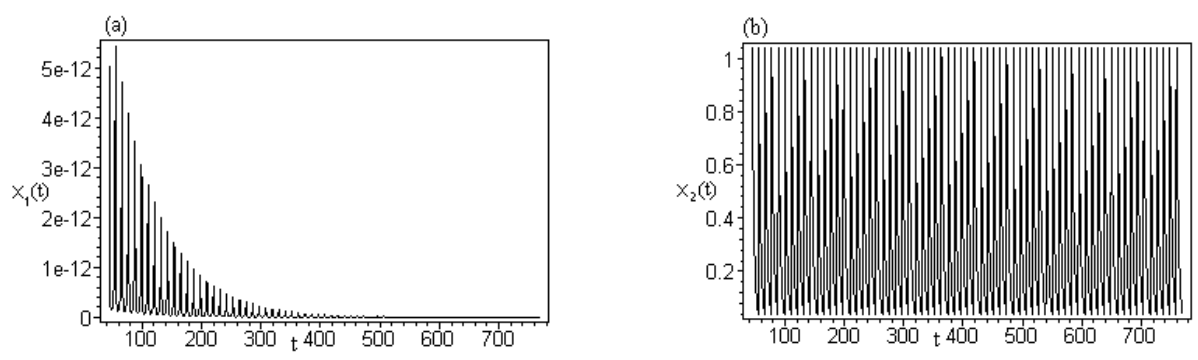

Figure 1. Dynamical behavior of the system (2.2) for $\tau=11$. (a) Time-series of the pest population. (b) Time-series of the natural enemy population.

$\frac{\partial \Theta_{2}}{\partial x_{2}}=1-c, \frac{\partial \Theta_{1}}{\partial x_{1}}=1, \frac{\partial^{2} \Theta_{2}}{\partial x_{1} \partial x_{2}}=0, \frac{\partial^{2} \Theta_{2}}{\partial x_{2}^{2}}=0$. Then

$$
\begin{aligned}
C= & 2(1-c) \frac{b_{0}^{\prime}}{a_{0}^{\prime}} \frac{\partial^{2} \Phi_{2}\left(\tau_{0}, x_{0}\right)}{\partial x_{1} \partial x_{2}}-(1-c) \frac{\partial^{2} \Phi_{2}\left(\tau_{0}, x_{0}\right)}{\partial x_{2}^{2}}>0 \\
B= & -\frac{\partial^{2} \Theta_{2}}{\partial x_{1} \partial x_{2}}\left(\frac{\partial \Phi_{1}\left(\tau_{0}, x_{0}\right)}{\partial \bar{\tau}}+\frac{\partial \Phi_{1}\left(\tau_{0}, x_{0}\right)}{\partial x_{1}} \frac{1}{a_{0}^{\prime}} \frac{\partial \Theta_{1}}{\partial x_{1}} \frac{\partial \Phi_{1}\left(\tau_{0}, x_{0}\right)}{\partial \bar{\tau}}\right) \frac{\partial \Phi_{2}\left(\tau_{0}, x_{0}\right)}{\partial x_{2}} \\
& -\frac{\partial^{2} \Theta_{2}}{\partial x_{2}}\left(\frac{\partial^{2} \Phi_{2}\left(\tau_{0}, x_{0}\right)}{\partial \bar{\tau} \partial x_{2}}+\frac{\partial^{2} \Phi_{2}\left(\tau_{0}, x_{0}\right)}{\partial x_{1} \partial x_{2}} \frac{1}{a_{0}^{\prime}} \frac{\partial \Theta_{1}}{\partial x_{1}} \frac{\partial \Phi_{1}\left(\tau_{0}, x_{0}\right)}{\partial \bar{\tau}}\right) \\
= & -\left(a_{10}-a_{12} d^{m} \frac{\exp \left(-a_{20} m \tau_{0}\right)}{\left(1-\exp \left(-a_{20} \tau_{0}\right)\right)^{m}}\right)-\frac{1-c}{a_{0}^{\prime}} \frac{\partial^{2} \Phi_{2}\left(\tau_{0}, x_{0}\right)}{\partial x_{1} \partial x_{2}} \frac{\partial \Phi_{1}\left(\tau_{0}, x_{0}\right)}{\partial \bar{\tau}} .
\end{aligned}
$$

To determine the sign of $B$, let $f(t)=a_{10}-a_{12} d^{m} \frac{\exp \left(-a_{20} m t\right)}{\left(1-\exp \left(-a_{20} \tau_{0}\right)\right)^{m}}$, then $f^{\prime}(t)=m a_{12} a_{20} d^{m} \frac{\exp \left(-a_{20} m t\right)}{\left(1-\exp \left(-a_{20} \tau_{0}\right)\right)^{m}}>0$, so $f(t)$ is strictly increasing. Since $\int_{0}^{\tau_{0}} f(t) \mathrm{d} t=\ln \frac{1}{1-c}>0$, we conclude that $f\left(\tau_{0}\right)>0$, from (5.1) we have $B<0$, thus $B C<0$. In view of $\tau_{0}=\tau_{\max }$ and Theorem 2 in [15], the following theorem holds true.

Theorem 5.1. Assume that $\tau_{\max }$ is the root of

$$
(1-c) \exp \left(a_{10} \tau_{0}-\frac{a_{12} d^{m}}{a_{20} m} \frac{1-\exp \left(-a_{20} m \tau_{0}\right)}{\left(1-\exp \left(-a_{20} \tau_{0}\right)\right)^{m}}\right)=1,
$$

then system (2.2) has a locally stable positive periodic solution when $\tau>\tau_{\max }$ and is close to $\tau_{\max }$.

\section{Numerical Result}

In this section, we take $a_{10}=1, a_{11}=0.6, a_{12}=3, a_{20}=0.3, c=0.2, d=1, m=0.9, k=2$, then $\tau_{\max }=11.13$. When period of pulses is less than critical value $\tau_{\max }$, there exists a pest-eradication periodic solution for the system (2.2). A typical pest-eradication periodic solution is shown in Figure 1, where we observe variable $x_{2}(t)$ oscillates in a stable cycle. In contrast, variable $x_{1}(t)$ rapidly reduces to zero. If period of pulses is larger than $\tau_{\max }=11.13$, pest-eradication periodic solution becomes unstable and natural enemies and pests coexist stably which corresponds to periodic burst of pest (see Fig. 2). If the period of pulses is further increased, the positive periodic solution loses stability and the model will exhibit complicated dynamical behaviors, including chaos. Figure $3 \mathrm{a}$ is the bifurcation diagram of the system (2.2) as impulsive period is the bifurcation parameter. It clearly shows a sequence of direct and inverse cascade of period-doubling (see Fig. 3b), chaotic bands, tangent bifurcation, periodic windows and crises.

When the parameter $\tau$ is slightly increased beyond $\tau=19.84$, the chaotic attractor abruptly appears, thus constituting a type of attractor crisis (the phenomenon of "crisis" in which chaotic attractor can suddenly appear 

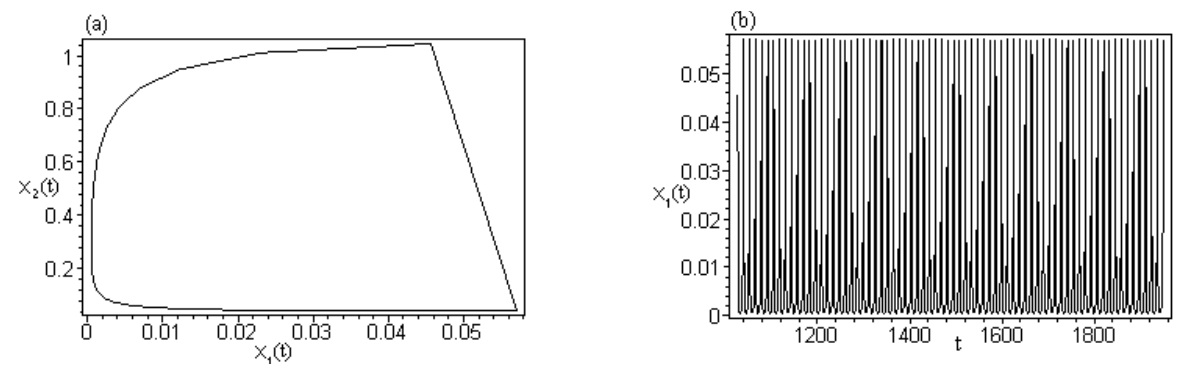

Figure 2. Coexistence of pest and natural enemy with initial value $\left(x_{1}\left(0^{+}\right), x_{2}\left(0^{+}\right)\right)=$ $(1.5,0.5)$ for $\tau=13$. (a) Phase portrait. (b) Time-series of pest population.
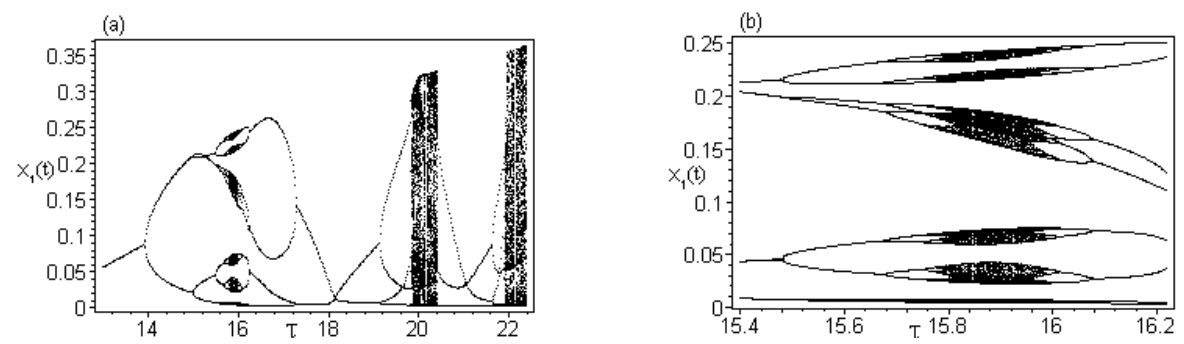

FiguRE 3. Bifurcation diagrams for the system $(2.2)$ with initial value $\left(x_{1}\left(0^{+}\right), x_{2}\left(0^{+}\right)\right)=$ $(1.5,0.5)$. (a) $x_{1}(t)$ are plotted with $\tau$ overing the range $[13,22.4]$. (b) The localy amplification of Figure 3a for $\tau \in[15.4,16.22]$.
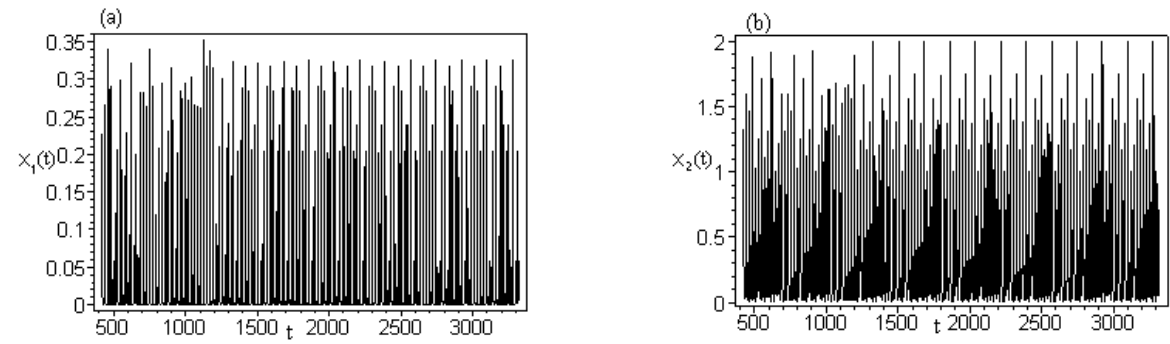

Figure 4. Supertransient of the system (2.2) for $\tau=22.14$. (a) Time-series of pest population.

(b) Time-series of natural enemy population.

or disappear, or change size discontinuously as a parameter smoothly varies, was first extensively analyzed by Grebogi et al. [6]. Crises also occur at $\tau=20.39, \tau=21.91$.

There are two interesting phenomena for the system (2.2), namely antimonotonicity and supertransients. Bifurcation diagram in Figure $3 \mathrm{a}$ shows cascades oriented in both directions. Thus the periodic orbits are both annihilated and created as the parameter $\tau$ is increased near certain common parameter values. This shows that the system (2.2) has the property of "antimonotonicity" which was studied by Dawson et al. [3]. Supertransients are used to denote an unusually long convergence to an attractor. These transient dynamics are considerably longer than the time-scale of significant environmental perturbations [6,11]. The time-scale of ecological interest is tens or hundreds time units, while supertransients can persist thousands time units or even longer. Figure 4 shows an example of supertransients. In this example, the pest and predator population size suddenly stabilizes into a periodic-seven attractor after 1294 time units of complicated fluctuations resembling 
an intermittent trajectory, respectively. Unfortunately, as the existence of supertransients implies, it maybe difficult to determine the appropriate and reliable length for a time series.

\section{Discussion}

In this paper, we discussed a Volterra model with mutual interference concerning integrated pest management. We have proved that there exists a globally asymptotically stable pest-eradication periodic solution when period of pulses is less than some critical value. When the stability of pest-eradication periodic solution is lost, we have shown that the system is permanent and there exists a locally stable positive periodic solution nearing the critical value, which is in line with reality from a biological point of view. Numerical results show there is a characteristic sequence of bifurcation, leading to chaotic dynamics, which implies that dynamical behaviors of Volterra model with mutual interference concerning IPM are very complex.

From Theorem 4.1, we know that $\tau_{\max }$ is a implicit function of $c$ and $d$. We can choose appropriate parameters $c$ and $d$ according to the effect of chemical pesticides on environment and cost of release natural enemies such that $\tau<\tau_{\max }$. That is, we can choose appropriate parameters $c$ and $d$ to eradicate pest with little cost and minimal effect on environment. In Figure 1 , we have $\tau_{\max }=11.13$, so we can make the impulsive periodic smaller than 11.13 in order to eradicate pest by choosing parameters $c$ and $d$.

Now, we can compare the validity of the IPM strategy with classical methods (only biological control or chemical control). If we only choose chemical control (i.e. $d=0$ ), with other parameters being the same as those of Figure 1, then we have $\tau_{\max }=0.22$. This implies that we must spray insecticides with much shorter time interval and we must use higher rates and more toxicant materials to combat pests since insect pests may quickly become resistant to pesticide, which does not accord with the objectives of IPM. If we only choose biological control (i.e. $c=0$ ), with other parameters being the same as those of Figure 1, then we have $\tau_{\max }=10.9$, which means we must release more natural enemies to eradicate pests. Therefore, IPM strategy is more effective than classical methods because it can both reduce cost and decrease pollution.

Acknowledgements. This work is supported by National Natural Science Foundation of China (10171106). We would like to thank the referee and the editor for their careful reading of the original manuscript.

\section{REFERENCES}

[1] D.D. Bainov and P.S. Simeonov, Impulsive differential equations: periodic solutions and applications. Wiley, New York (1993).

[2] A.J. Cherry, C.J. Lomer, D. Djegui and F. Schulthess, Pathogen incidence and their potential as microbial control agents in IPM of maize stemborers in West Africa. Biocontrol 44 (1999) 301-327.

[3] S.P. Dawson, C. Grebogi and J.A. Yorke, Antimonotonicity: inevitable reversals of period-doubling cascades. Phys. Lett. A 162 (1992) 249-254.

[4] R.L. Edwards, The area of discovery of two insect parasites, Nasonia vitripennis (Walker) and Trichogramma evanescens Westwood, in an artificial environment. Can. Ent. 93 (1961) 475-481.

[5] J. Grasman, O.A. Van Herwaarden, L. Hemerik et al., A two-component model of host-parasitoid interactions: determination of the size of inundative releases of parasitoids in biological pest control. Math. Biosci. 169 (2001) 207-216.

[6] C. Grebogi, E. Ott and J.A. York, Crises, sudden changes in chaotic attractors and chaotic transients. Physica D 7 (1983) $181-200$.

[7] M.P. Hassell, Parasite behavior as a factor contributing to the stability of insect host-parasite interactions, in Dynamics of Population, P.J. den Boer and G.R. Gradwell Eds., Proc. Adv. Study Inst. Oosterbeek (1970).

[8] M.P. Hassell, Mutual interference between searching insect parasites. J. Anim. Ecol. 40 (1971) 473-486.

[9] M.P. Hassell, G.C. Varley, New inductive population model for insect parasites and its bearing on biological control. Nature 223 (1969) 1133-1136.

[10] M.P. Hassell and D.J. Rogers, Insect parasite responses in the development of population models. J. Anim. Ecol. 41 (1972) $661-676$.

[11] A. Hasting and K. Higgins, Persistence of transients in spatially structured ecological models. Since 263 (1994) $1133-1136$.

[12] C.B. Huffaker, P.S. Messenger and P. De Bach, The natural enemy component in natural control and the theory of biological control, in Biological Control, C.B. Huffaker Ed., Proc. A.A.A.S. Symp. Plenum Press, New York (1969). 
[13] Integrated Pest Management for Walnuts, University of California Statewide Integrated Pest Management Project, in Division of Agriculture and Natural Resources, Second Edition, M.L. Flint Ed., University of California, Oakland, CA, publication 3270 (1987).

[14] V. Lakshmikantham, D.D. Bainov and P.S. Simeonov, Theory of impulsive differential equations. World Scientific, Singapore (1989).

[15] A. Lakmeche and O. Arino, Bifurcation of non trival periodic solutions of impulsive differential equations arising chemotherapeutic treatment. Dynam. Contin. Discrete Impuls. Systems 7 (2000) 205-287.

[16] M.L. Luff, The potential of predators for pest control. Agri. Ecos. Environ. 10 (1983) 159-181.

[17] D.J. Rogers, Random search and insect population models. J. Anim. Ecol. 41 (1972) 369-383.

[18] University of California, Division of Agriculture and Natural Resources, Integrated Pest Management for Alfafa hay. Publications, Division of Agriculture and Nature Resources, University of Califania, Oakland, CA, publication 3312 (1981).

[19] J.C. Van Lenteren, Integrated pest management in protected crops, in Integrated pest management, D. Dent Ed., Chapman \& Hall, London (1995).

[20] P.L. Wu, Stability of Volterra model with mutual interference. J. Jiangxi Norm. Univ. 2 (1986) 42-45.

To access this journal online: www.edpsciences.org 